\title{
THE EFFECTS OF A LOW-CALORIE DIET OR AN ISOCALORIC DIET COMBINED WITH METFORMIN ON SEX HORMONES IN OBESE WOMEN OF CHILD-BEARING AGE
}

\author{
Ewelina Swora-Cwynar ${ }^{1}$, Magdalena Kujawska-Łuczak², Joanna Suliburska ${ }^{3}$, \\ Julita Reguła3 ${ }^{3}$ Angelika Kargulewicz ${ }^{1}$, Matylda Kręgielska-Narożna ${ }^{4}$, \\ Emilia Marcinkowska ${ }^{1}$ Alina Kanikowska¹, Marzena Bielas², \\ Marian Grzymisławski ${ }^{1}$, Paweł Bogdański ${ }^{4 \bowtie}$
}

\author{
${ }^{1}$ Department of Internal Medicine, Metabolic Disorders and Dietetics, Poznan University of Medical Sciences \\ Przybyszewskiego 49, 60-355 Poznań, Poland \\ 2Department of Internal Medicine, Metabolic Disorders, and Hypertension, Poznan University of Medical Sciences \\ Szamarzewskiego 84, 60-569 Poznań, Poland \\ ${ }^{3}$ Department of Human Nutrition and Hygiene, Poznań University of Life Sciences \\ Wojska Polskiego 31, 60-624 Poznań, Poland \\ ${ }^{4}$ Department of Education and Obesity Treatment and Metabolic Disorders, Poznan University of Medical Sciences \\ Szamarzewskiego 84, 60-569 Poznań, Poland
}

\begin{abstract}
Background. The influence of weight loss treatment on sex hormones profile has been studied mainly in women with polycystic ovary syndrome (PCOS), but in obese premenopausal women without PCOS it still remains unclear. The aim of the study was to evaluate the effect of two approaches to obesity treatment on the serum level of sex hormones in obese women of child-bearing age without PCOS.

Material and methods. 77 obese Caucasian women (aged $31.2 \pm 8.3$ years) were randomized into two groups: 39 women received a low-calorie diet (LC) and 38 received an isocaloric diet plus metformin (IM), for 12 weeks. Anthropometric parameters, body composition and serum concentrations of estradiol (E2), testosterone (T), follicle-stimulating hormone (FSH), luteinizing hormone ( $\mathrm{LH}$ ) and dehydroepiandrosterone (DHEA-S) sulfate were evaluated at baseline and after the study.

Results. Reductions in body weight, body mass index (BMI), waist and body fat content with an increase in lean body percent were significant and comparable between the LC and IM group after the trial. The concentrations of serum FSH, LH, E2, DHEA and T did not change in either group after treatment. A tendency towards an increase in the E2 concentration in both groups and a decrease in the T level in the LC group was observed. The correlations between a change in BMI, fat content, waist-hip ratio and a change in $\mathrm{T}$ were documented in the LC group.

Conclusion. A 12-week low-calorie diet and an isocaloric diet combined with metformin produced comparable and significant weight loss with improvements in body composition. Both interventions did not significantly affect FSH, LH and DHEA sulfate serum concentrations, only a trend towards an E2 increase and a T decrease was observed, stronger in LC group. The significant correlations shown between the changes in anthropometric and body composition parameters and $\mathrm{T}$ serum levels in women treated with a low-calorie diet alone show the beneficial effect of a lifestyle intervention on the sex hormone in obese premenopausal women.
\end{abstract}

Key words: obesity, sex hormones, metformin, low-calorie diet

pawelbogdanski@wp.pl 

with metformin on sex hormones in obese women of child-bearing age. Acta Sci. Pol. Technol. Aliment., 15(2), 213-220. DOI: 10.17306/J.AFS.2016.2.21

\section{INTRODUCTION}

Obesity in women of childbearing age is becoming an increasingly frequent problem associated with metabolic complications, including the effect on the endocrine system and sex hormone concentrations. Adipose tissue and sex hormones play an active role in mutual regulation and metabolism. Elevated levels of androgens in women and decreased levels in men predispose to the accumulation of adipose tissue within the abdominal cavity and result in the higher risk of developing metabolic diseases. Androgens stimulate the reduction of adipose tissue and increase lean body mass in men. In women, estrogens promote the deposition of fat in the gluteofemoral region, which accounts for a significantly lower risk of developing metabolic complications when compared to a more visceral localization of adipose tissue (Escobar-Moreale et al., 2014). The relation between serum dehydroepiandrosterone (DHEA) and the amount and distribution of adipose tissue is not fully defined. The concentration of DHEA decreases with age and depends on sex, degree of obesity, health status, and many other factors. Higher concentrations of DHEA were observed in women with lower body mass index (BMI) values. Negative correlations between serum DHEA concentration and waist circumference and serum DHEA and waist-hip ratio (WHR) accompanied by a positive correlation between serum DHEA and testosterone (T) levels were found in women with polycystic ovary syndrome (PCOS) (Chen et al., 2011). It was observed that women with a lower caloric intake had higher concentrations of DHEA; on the contrary, those with a higher caloric intake showed lower concentrations of DHEA, which may indicate that DHEA has an effect on appetite regulation (Michalakis et al., 2013). The study by Kurtz et al. showed that there was an increased turnover of DHEA in obese women. A significant positive correlation was observed between abdominal obesity and an increased clearance of DHEA and androstendione, which suggests that the androgenic effect of DHEA may influence the distribution of adipose tissue (Demissie and Milewicz 2003; Kurtz et al., 1987). Decreased concentrations of luteinizing hormone (LH) and follicle-stimulating hormone (FSH) were found in some studies in obese women compared to normal-weight women, although other authors did not describe such a correlation. Obese women also had a decreased concentration of circulating sex hormone-binding globulin (SHBG), and consequently an increased fraction of circulating estradiol (E2). In postmenopausal women, estrogen levels (E1 and E2) correlate with the degree of obesity and the amount of adipose tissue. Moreover, they inhibit the secretion of gonadotropins (Ivandic et al., 1998).

Depending on its serum concentration, testosterone has different effects. Moderately increased concentrations stimulate the synthesis of LH, while high concentrations have the opposite effect. Several studies have shown that women with abdominal obesity have higher levels of T and E2 compared to women with gluteofemoral obesity (Rosenfeld and Bordini, 2010).

The main treatment for obesity involves the use of an optimal diet combined with regular exercise. Antiobesity medications including metformin may additionally correct concomitant hormonal and metabolic disturbances. The fact that obesity interferes with the endocrine system in women makes the treatment capable of bringing promising health benefits to the sex hormone profile of obese women.

The aim of this study was to evaluate the effect of a low-calorie diet versus an isocaloric diet combined with metformin on sex hormone levels in obese premenopausal women during a 12-week randomized prospective open-label trial.

\section{MATERIAL AND METHODS}

\section{Study design and patients}

The study was approved by the Ethics Committee of Poznan University of Medical Sciences, case no. $688 / 09$. The trial protocol met the requirements of the Declaration of Helsinki. Out of the registered patients screened at the outpatient clinic of the Department of Internal Medicine, Metabolism, and Dietetics and the Department of Internal Medicine, Metabolic Disorders, and Hypertension of Poznan University of Medical Sciences, Poland a total of 77 obese women were enrolled. Informed consent was obtained from all individual participants included in the study.

The inclusion criteria were as follows: age -18 to 40 years, obesity - BMI $\geq 30 \mathrm{~kg} / \mathrm{m}^{2}$; stable body weight for one month prior to the trial (acceptable deviation was $\pm 1 \mathrm{~kg}$ ), Caucasian race. 

with metformin on sex hormones in obese women of child-bearing age. Acta Sci. Pol. Technol. Aliment., 15(2), 213-220. DOI: 10.17306/J.AFS.2016.2.21

To achieve the homogeneity of participants the following exclusion criteria were accepted: secondary obesity; diabetes mellitus; $2^{\text {nd }}$ and $3^{\text {rd }}$ stage of hypertension; coronary artery disease; clinically significant arrhythmias or conduction disorders; congestive heart failure; stroke; malignancy; history of use of any dietary supplements in the month prior to the study; abnormal kidney or thyroid gland function; clinically significant acute or chronic inflammatory processes; infection in the month prior to the study; nicotine, alcohol, or drug abuse; pregnancy or childbirth at enrollment or in the 3 months prior to enrollment; current lactation or lactation in the 3 months prior to enrollment; PCOS, menopause; and any other condition that, in the opinion of the researchers, would render participation against the subject's best interests.

The study was designed as a prospective randomized open-label trial with comparison of a lowcalorie diet versus an isocaloric diet combined with metformin treatment. All women underwent a 12-week run-in period, which started with them receiving dietary advice from a qualified dietician, where they were counselled to adhere to a weightmaintenance diet. $25 \%$ of total energy comprised of fat, $20-25 \%$ protein and $50-55 \%$ carbohydrates (Table 1). Dietary intake was monitored every 14 days until the end of the trial by a qualified dietitian on the basis of dietary intake interviews and food diaries.

Table 1. Diet composition

\begin{tabular}{lc}
\hline \multicolumn{1}{c}{ Diet component } & $\begin{array}{c}\text { Percentage of total } \\
\text { energy intake }\end{array}$ \\
\hline Carbohydrate intake including: & $50-55$ \\
- complex carbohydrates & $45-50$ \\
- saccharose & $<10$ \\
Protein intake & $20-25$ \\
Fat intake including: & 25 \\
- saturated fatty acids & 7 \\
- monounsaturated fatty acids & 10 \\
- polyunsaturated fatty acids & 8 \\
Cholesterol intake, mg/d & $<300$ \\
\hline
\end{tabular}

The intake of nutrients, total calories, and caffeine was maintained at a constant level during the study. The selection of food was arranged individually with subjects. During the trial, consumption of dietary supplements was not permitted.

After the run-in period all women underwent a 12 week-long intervention aimed at reducing their body weight. Participants $(n=77)$ aged $31.4 \pm 8.2$ years were randomized into two study groups: LC $(n=39)$ received a low-calorie diet for 12 weeks and group IM $(n=38)$ received an individual isocaloric diet combined with the administration of metformin at $500 \mathrm{mg}$ twice a day for 12 weeks. All subjects were instructed to maintain their current physical activity at an unchanged level.

\section{Anthropometric and body composition measurements}

Anthropometric parameters. Anthropometric indices were measured while the subjects were barefoot and wearing light clothing. Height was measured to the nearest $0.5 \mathrm{~cm}$ and weight to the nearest $0.1 \mathrm{~kg}$. BMI was calculated as weight in kilograms divided by height in meters squared $\left[\mathrm{kg} / \mathrm{m}^{2}\right]$. Obesity was diagnosed with a BMI $\geq 30 \mathrm{~kg} / \mathrm{m}^{2}$. Waist and hip circumferences were measured to the nearest $0.5 \mathrm{~cm}$. Hip circumference was measured at the maximum protuberance of the buttocks. Waist circumference was measured at midway between the uppermost border of the iliac crest and the lower border of the costal margin at the end of normal expiration. The waist-hip ratio (WHR) was calculated as waist circumference divided by hip circumference.

Analysis of bio-impedance. Body composition was determined using bioelectrical impedance analysis with a Bodystat analyzer (1500 MDD; Bodystat, Isle of Man, UK). The analysis was performed under stable laboratory conditions after a night's rest.

Sex hormone analyses. The subjects' plasma were analysed. Samples of venous blood were drawn from a forearm vein after a 14-hour overnight fast (drinking of water was permitted) at the beginning and 12 weeks after the start of the trial. Biochemical parameters were estimated by electrochemiluminescent immunoassay: estradiol (E2) (ECLIA, Roche Diagnostic, Mannheim, 
Germany), testosterone (T) (ECLIA, Roche Diagnostic, Mannheim, Germany), luteinizing hormone (LH) (ECLIA, Roche Diagnostic, Mannheim, Germany), follicle-stimulating hormone (FSH) (ECLIA, Roche Diagnostic, Mannheim, Germany), dehydroepiandrosterone (DHEAS) sulfate (ECLIA, Roche Diagnostic, Mannheim, Germany).

Statistical analysis. The data was presented as arithmetic mean and standard deviation. All calculations and demographics were performed with STATISTICA 10 software (StatSoft, 2011). Comparison between groups was performed using the Mann-Whitney $U$ test. The Wilcoxon rank sum test was used to analyze the statistical significance between variables before and after the 12-week intervention. Simple associations between variables were calculated with the Spearman coefficient of correlation. A $p$-value of less than 0.05 was regarded as significant. It was determined that a sample size of a minimum 30 subjects in each group would yield at least a $80 \%$ probability of detecting an effect statistically significant at the $0.05 \alpha$ level.

\section{RESULTS}

There were no statistically significant differences prior to the study between the two randomized groups regarding all parameters studied.

The significant weight reduction $(p<0.001)$ was observed in both the low-calorie diet (LC) and isocaloric diet + metformin (IM)-receiving groups after the study. BMI decreased in both groups after the treatment, with values of high statistical significance for the IM group ( $p<0.001$ for IM versus $p<0.01$ for LC group). Waist reduction was statistically significant to a high degree in the LC group $(p<0.001)$ and statistically significant in the IM group $(p<0.05)$, with a comparison of change $(\Delta)$ results between groups regarding the waist also being statistically significant $(p<0.05)$.

Both types of intervention led to a significant decrease in fat content and an increase in lean the tissue percentage in obese women from the LC and IM groups, although the comparison of $\Delta$ results between the groups did not reveal any statistical significance.

Table 2. Anthropometric indices and body composition before and after treatment

\begin{tabular}{|c|c|c|c|c|c|c|c|}
\hline & \multicolumn{3}{|c|}{$\begin{array}{l}\text { Low-calorie diet (LC) } \\
\text { group }\end{array}$} & \multicolumn{3}{|c|}{$\begin{array}{l}\text { Isocaloric diet }+ \text { metformin (IM) } \\
\text { group }\end{array}$} & \multirow{2}{*}{$\begin{array}{c}\text { Comparison of } \\
\text { change }(\Delta) \text { results } \\
\text { between groups } \\
p \text {-value }\end{array}$} \\
\hline & before & after & $p$-value & before & after & $p$-value & \\
\hline Weight, kg & $100.6 \pm 16.4$ & $96.0 \pm 14.3$ & $<0.001^{*}$ & $108.8 \pm 24.0$ & $103.7 \pm 23.7$ & $<0.001 *$ & NS \\
\hline $\begin{array}{l}\text { Body mass index } \\
-\mathrm{BMI}, \mathrm{kg} / \mathrm{m}^{2}\end{array}$ & $36.1 \pm 5.6$ & $34.5 \pm 4.9$ & $<0.01^{*}$ & $40.1 \pm 8.1$ & $38.1 \pm 7.9$ & $<0.001^{*}$ & NS \\
\hline Waist, cm & $104.5 \pm 12.9$ & $96.3 \pm 10.6$ & $<0.001^{*}$ & $109.6 \pm 16.0$ & $105.1 \pm 16.6$ & $<0.05^{*}$ & $<0.05$ \\
\hline Waist-hip ratio - WHR & $0.82 \pm 0.07$ & $0.81 \pm 0.07$ & NS & $0.87 \pm 0.12$ & $0.84 \pm 0.08$ & NS & NS \\
\hline Fat, \% & $46.6 \pm 5.6$ & $44.8 \pm 5.3$ & $<0.001^{*}$ & $46.6 \pm 6.2$ & $44.3 \pm 5.8$ & $<0.001 *$ & NS \\
\hline Fat, kg & $47.2 \pm 10.8$ & $42.4 \pm 11.5$ & $<0.01^{*}$ & $47.2 \pm 15.9$ & $42.9 \pm 15.4$ & $<0.001^{*}$ & NS \\
\hline Lean, \% & $55.3 \pm 2.5$ & $57.6 \pm 3,7$ & $<0.01^{*}$ & $55.0 \pm 5.6$ & $57.2 \pm 5.7$ & $<0.01^{*}$ & NS \\
\hline Lean, kg & $56.8 \pm 9.7$ & $56.3 \pm 7.0$ & NS & $55.6 \pm 6.4$ & $55.1 \pm 6.5$ & NS & NS \\
\hline
\end{tabular}

Data are presented as arithmetic mean \pm standard deviation.

*Refer to statistically significant differences $(p<0.05)$ between initial and final values.

NS - not statistically significant. 
Swora-Cwynar, E., Kujawska-Łuczak, M., Suliburska, J., Reguła, J., Kargulewicz, A., Kręgielska-Narożna, M., Marcinkowska, E., Kanikowska, A., Bielas, M., Grzymisławski, M., Bogdański, P. (2016). The effects of a low-calorie diet or an isocaloric diet combined with metformin on sex hormones in obese women of child-bearing age. Acta Sci. Pol. Technol. Aliment., 15(2), 213-220. DOI: 10.17306/J.AFS.2016.2.21

Table 3. Results of hormonal tests, before and after treatment

\begin{tabular}{|c|c|c|c|c|c|c|c|}
\hline & \multicolumn{3}{|c|}{ Low-calorie diet (LC) group } & \multicolumn{3}{|c|}{ Isocaloric diet + metformin (IM) group } & \multirow{2}{*}{$\begin{array}{c}\text { Comparison of } \\
\text { change }(\Delta) \text { results } \\
\text { between groups } \\
p \text {-value }\end{array}$} \\
\hline & before & after & $p$-value & before & after & $p$-value & \\
\hline Estradiol-E2,pg/ml & $89.0 \pm 64.8$ & $111.1 \pm 116.3$ & NS & $52.7 \pm 52.1$ & $92.0 \pm 152.4$ & NS & NS \\
\hline $\begin{array}{l}\text { Testosterone }-\mathrm{T} \\
\mathrm{nmol} / 1\end{array}$ & $1.45 \pm 0.48$ & $1.15 \pm 0.56$ & NS & $1.36 \pm 0.92$ & $1.40 \pm 0.90$ & NS & NS \\
\hline $\begin{array}{l}\text { Luteinizing hor- } \\
\text { mone - LH, mlU/ml }\end{array}$ & $7.05 \pm 4.53$ & $7.01 \pm 4.13$ & NS & $6.98 \pm 8.92$ & $5.77 \pm 4.31$ & NS & NS \\
\hline $\begin{array}{l}\text { Follicle-stimulating } \\
\text { hormone - FSH } \\
\mathrm{mlU} / \mathrm{ml}\end{array}$ & $4.74 \pm 1.90$ & $5.66 \pm 2.38$ & NS & $4.68 \pm 2.73$ & $4.76 \pm 2.58$ & NS & NS \\
\hline $\begin{array}{l}\text { Dehydroepiandros- } \\
\text { terone sulfate } \\
\text { - DHEAS, } \mu \mathrm{g} / \mathrm{dl}\end{array}$ & $296.3 \pm 123.4$ & $249.6 \pm 133.8$ & NS & $259.4 \pm 117.6$ & $262.2 \pm 88.7$ & NS & NS \\
\hline
\end{tabular}

Data are presented as arithmetic mean \pm standard deviation.

NS - not statistically significant.

Detailed values of the anthropometric and body composition parameters in the womenstudied before and after treatment are summarized in Table 2.

Neither a low-calorie diet nor nor an isocaloric diet + metformin treatment were able to affect E2, T, LH, FSH and DHEA sulfate serum hormone levels significantly in obese women. However, a non-significant tendency for an increase in E2 concentration in both groups and a decrease of T level in the LC group was noticed (Table 3).

Statistically significant positive correlations between the $\Delta \mathrm{T}$ serum level and $\Delta \mathrm{BMI}(r=0.55)$, $\Delta$ WHR $(r=0.57)$ and $\Delta$ fat content $(r=0.64)$ were only documented in women from the LC group. Correlations between $\Delta$ in anthropometric parameters and $\Delta$ sex hormone levels in population studied are presented in Table 4.

Table 4. Correlation between changes $(\Delta)$ in serum sex hormone concentrations and changes $(\Delta)$ in anthropometric parameters in groups

\begin{tabular}{lcccccccc}
\hline & \multicolumn{3}{c}{ Low-calorie diet (LC) group } & \multicolumn{3}{c}{ Isocaloric diet + metformin (IM) group } \\
\cline { 2 - 9 } & $\begin{array}{c}\Delta \mathrm{BMI} \\
\mathrm{kg} / \mathrm{m}^{2}\end{array}$ & $\Delta$ WHR & $\begin{array}{c}\Delta \text { Fat } \\
\%\end{array}$ & $\begin{array}{c}\Delta \text { Fat } \\
\mathrm{kg}\end{array}$ & $\begin{array}{c}\Delta \text { BMI } \\
\mathrm{kg} / \mathrm{m}^{2}\end{array}$ & $\Delta$ WHR & $\begin{array}{c}\Delta \text { Fat } \\
\%\end{array}$ & $\begin{array}{c}\Delta \text { Fat } \\
\mathrm{kg}\end{array}$ \\
\hline$\Delta \mathrm{E} 2, \mathrm{pg} / \mathrm{ml}$ & 0.24 & -0.30 & 0.02 & 0.16 & 0.37 & -0.10 & 0.22 & 0.36 \\
$\Delta \mathrm{T}, \mathrm{nmol} / \mathrm{l}$ & $0.55^{*}$ & $0.57^{*}$ & 0.21 & $0.64^{*}$ & 0.09 & 0.10 & -0.04 & 0.02 \\
$\Delta \mathrm{LH}, \mathrm{mlU} / \mathrm{ml}$ & 0.31 & 0.02 & -0.09 & 0.16 & -0.23 & -0.18 & -0.04 & 0.01 \\
$\Delta \mathrm{FSH}, \mathrm{mlU} / \mathrm{ml}$ & 0.52 & 0.26 & -0.02 & 0.47 & -0.17 & 0.30 & 0.05 & 0.08 \\
$\Delta \mathrm{DHEAS}, \mu \mathrm{g} / \mathrm{dl}$ & -0.14 & 0.33 & 0.35 & 0.21 & -0.01 & 0.43 & 0.14 & 0.16 \\
\hline
\end{tabular}

*Refer to statistically significant correlations $(p<0.05)$.

BMI - body mass index, WHR - waist-hip ratio, FSH - follicle-stimulating hormone, LH - luteinizing hormone, E2 - estradiol, DHEAS - dehydroepiandrosterone sulfate, $\mathrm{T}$ - testosterone. 

with metformin on sex hormones in obese women of child-bearing age. Acta Sci. Pol. Technol. Aliment., 15(2), 213-220. DOI: 10.17306/J.AFS.2016.2.21

\section{DISCUSSION}

A low-calorie diet and physical activity are standard approaches recommended for the treatment of obesity. Metformin, used increasingly in obesity treatment, is believed to exert a beneficial effect on comorbid metabolic and hormonal disturbances.

Our study showed that regardless of the type of intervention: a low-calorie diet or an isocaloric diet combined with metformin, the levels of sex hormones including E2, DHEA, LH, FSH and T in obese women of childbearing age remained almost unchanged. Women from both the LC and IM group achieved a significant reduction in body weight, waist circumference and body fat content and an increase in lean body mass. There was a positive correlation between $\Delta \mathrm{BMI}, \Delta \mathrm{WHR}$ and $\Delta$ fat content and a change in $\mathrm{T}$ levels in the group treated with the low-calorie diet alone.

Animal studies showed that weight gain and increased abdominal adipose tissue observed in rats after ovariectomy can be reduced by treatment with estrogens or phytoestrogens (Rachoń and Teede, 2010). In our study, both the LC and IM group of obese women revealed a nonsignificant tendency for an increase in E2 concentration after the 12-week intervention period. In the study by Al-Nozha et al. (2013) performed on overweight PCOS women, after a weight reduction was achieved, an analogous tendency for an increase in E2 concentrations was detected. In the other study, a similar trend or statically significant difference in E2 concertation was documented after 7 months of a hypocaloric diet + metformin treatment, in obese PCOS and obese women, respectively (Pasquali et al., 2000). An inverse relation between estradiol and body mass (and BMI) was documented by others with respect to postmenopausal women (Jones et al., 2013). Estradiol production in menopausal women is closely associated with adipose tissue. It has been shown that changes in BMI in postmenopausal overweight women were related to a significant decrease in the level of estrogen (Jones et al., 2013).

The significant reduction in weight was observed in both LC and IM groups. BMI decreased in LC and IM women with values with high statistical significance for the IM group. Pasquali et al. (2000) also reported changes in body weight and BMI in premenopausal obese women and women with PCOS, during hypocaloric dieting and after 6 months of pharmacological treatment with metformin.

The association between reduction in BMI and a decrease in the serum $T$ level in obese postmenopausal or PCOS women receiving a reduced-calorie diet was observed in several studies (Campbell et al., 2012; Eid et al., 2014; Gemert et al., 2015; Jones et al., 2013). The positive effect of metformin on the reduction in $\mathrm{T}$ and androstenedione concentrations in women with BMI $>25 \mathrm{~kg} / \mathrm{m}^{2}$ was detected at a dose of $1000-1700 \mathrm{mg} /$ day, as compared with a low-calorie diet (1500 kcal) used alone (Ghandi et al., 2011; Jayagopal et al., 2005).

In our study, a positive correlation between weight reduction (a decrease in BMI, WHR and fat content) and the concentration of $\mathrm{T}$ in serum was found in premenopausal women on a low-calorie diet. Furthermore, a statistically non-significant trend towards a decrease in the $\mathrm{T}$ concentration in the LC group was noticed. However, we did not observe any influence of metformin combined with an isocaloric diet on the $\mathrm{T}$ concentration in the women studied. In contrast to our results, Pasquali et al. (2000) demonstrated an association between a reduction in body weight and abdominal adipose tissue with a decrease in the T serum level in women after 6 months' treatment with $1700 \mathrm{mg}$ metformin/day, combined with a low-calorie diet of 1200-1400 kcal/day. The authors demonstrated a lower level of $\mathrm{T}$ in obese women and obese women with PCOS after a low calorie diet + metformin or a low calorie diet + placebo treatment. Weight loss combined with the use of metformin did not affect the concentrations of DHEAS, LH or FSH in this study, which was similar to our results. In another study, women with PCOS treated with hormonal therapy using clomiphene citrate were divided into three groups: those with no additional intervention, those treated with a low-calorie diet, and those treated with metformin. After 6 months, all groups showed a statistically significant decrease in lipid, insulin, T, FSH/LH, SHBG and PRL levels. There was a positive correlation between abdominal obesity, increased arm circumference and serum $\mathrm{T}$ levels. The greatest reduction in body weight was observed in the group treated with a low-calorie diet, a slightly lower reduction in the group treated with metformin and the smallest reduction in body weight was observed in the group of 
Swora-Cwynar, E., Kujawska-Łuczak, M., Suliburska, J., Reguła, J., Kargulewicz, A., Kręgielska-Narożna, M., Marcinkowska, E., Kanikowska, A., Bielas, M., Grzymisławski, M., Bogdański, P. (2016). The effects of a low-calorie diet or an isocaloric diet combined with metformin on sex hormones in obese women of child-bearing age. Acta Sci. Pol. Technol. Aliment., 15(2), 213-220. DOI: 10.17306/J.AFS.2016.2.21

patients only receiving hormonal therapy. The androgen-reducing effect may be caused by a reduction in body weight and metformin may have a slight additive effect on it when compared to the effect resulting from diet treatment on the reduction in body weight (Al-Nozha et al., 2013).

Unquestionably, this study has some limitations: firstly, in our study we did not have a group of women treated with only metformin. We tested only dietary weight loss vs. one dose of metformin. Secondly, our study was designed as a relatively short one (12 weeks) with a low dose of metformin $(1000 \mathrm{mg})$. Thirdly, we did not analyze the impact of other factors affecting the levels of sex hormones, e.g. distribution of adipose tissue, duration of obesity, duration of intervention.

In conclusion, in obese women of child-bearing age both interventions: a 12-week low-calorie diet and an isocaloric diet combined with metformin produce comparable and significant weight loss with improvements in body composition. However, both interventions do not significantly affect FSH, LH and DHEA sulfate serum concentrations. The relationship between a reduction in body weight, fat content and a reduction in $\mathrm{T}$ serum level in the participants studied who were receiving a low-calorie diet alone suggests the beneficial influence of a dietary intervention approach on the regulation of sex hormone levels in obese women of child-bearing age without PCOS.

\section{REFERENCES}

Al-Nozha, O., Habib, F., Mojaddidi, M., El-Bab, M. F. (2013). Body weight reduction and metformin: Roles in polycystic ovary syndrome. Pathophysiology, 20(2), 131-137.

Campbell, K. L., Foster-Schubert, K. E., Alfano, C. M., Wang, C. C., Wang, C. Y., Duggan, C.R., ..., McTiernan, A. (2012). Reduced-calorie dietary weight loss, exercise, and sex hormones in postmenopausal women: randomized controlled trial. J. Clin. Oncol., 30(19), 2314-2326.

Chen, M. J., Chen, C. D., Yang, J. H., Chen, C. L., Ho, H. N., ..., Yang, W. S. (2011). High serum dehydroepiandrosterone sulfate is associated with phenotypic acne and a reduced risk of abdominal obesity in women with polycystic ovary syndrome. Hum. Reprod., 26(1), 227-234.
Demissie, M., Milewicz, A. (2003). Hormonal disturbances in obesity. Diabet. Prakt., 4(3), 207-209.

Eid, G. M., McCloskey, C., Titchner, R., Korytkowski, M., Gross, D., Grabowski, C., Wilson, M. (2014). Changes in hormones and biomarkers in polycystic ovarian syndrome treated with gastric bypass. Surg. Obes. Relat. Dis., 10(5), 787-791.

Escobar-Morreale, H. F., Alvarez-Blasco, F., Botella-Carretero, J. I., Luque-Ramírez, M. (2014). The striking similarities in the metabolic associations of female androgen excess and male androgen deficiency. Hum. Reprod., 29(10), 2083-2091.

Gemert van, W. A., Schuit, A. J., van der Palen, J., May, A. M., Iestra, J. A., Wittink, H., ..., Monninkhof, E. M. (2015). Effect of weight loss, with or without exercise, on body composition and sex hormones in postmenopausal women: the SHAPE-2 trial. Breast Cancer Res., 2, 17(1), 120 .

Ghandi, S., Aflatoonian, A., Tabibnejad, N., Moghaddam, M. H. (2011). The effects of metformin or orlistat on obese women with polycystic ovary syndrome: a prospective randomized open-label study. J. Assist. Reprod. Genet., 28(7). 591-596.

Ivandic, A., Prpic-Krizevac, I., Sucic, M., Juric, M. (1998). Hyperinsulinemia and sex hormones in healthy premenopausal women: relative contribution of obesity, obesity type and duration of obesity. Metabolism, 47(1), 13-19.

Jayagopal, V., Kilpatrick, E. S., Holding, S., Jennings, P. E., Atkin, S. L. (2005). Orlistat is as beneficial as metformin in the treatment of polycystic ovarian syndrome. J. Clin. Endocrinol. Metab., 90(2), 729-733.

Jones, M. E., Schoemaker, M., Rae M., Folkerd, E. J., Dowsett, M., Ashworth, A., Swerdlow, A. J. (2013). Changes in estradiol and testosterone levels in postmenopausal women after changes in body mass index. J. Clin. Endocrinol. Metab., 98(7), 2967-2974.

Kelly, C. J., Gordon, D. (2002). The effect of metformin on hirsutism in polycystic ovary syndrome. Eur. J. Endocrinol., 147(2), 217-221.

Kurtz, B. R., Givens, J. R., Komindr, S., Stevens, M. D., Karas, J. G., Bittle, J. B., ..., Judge, D. (1987). Maintenance of normal circulating levels of androstendione and dehydroepiandrosterone in simple obesity despite increased metabolic clearance rates. J. Clin. Endocrinol. Metab., 64(6), 1261-1267.

Michalakis, K., Mintziori, G., Kaprara, A., Tarlatzis, B. C., Goulis, D. G. (2013). The complex interaction between obesity, metabolic syndrome and reproductive axis: a narrative review. Metabolism, 62(4), 457-478. 
Swora-Cwynar, E., Kujawska-Łuczak, M., Suliburska, J., Reguła, J., Kargulewicz, A., Kręgielska-Narożna, M., Marcinkowska, E., Kanikowska, A., Bielas, M., Grzymisławski, M., Bogdański, P. (2016). The effects of a low-calorie diet or an isocaloric diet combined with metformin on sex hormones in obese women of child-bearing age. Acta Sci. Pol. Technol. Aliment., 15(2), 213-220. DOI: 10.17306/J.AFS.2016.2.21

Pasquali, R., Gambineri, A., Biscotti, D., Vicennati, V., Gagliardi, L., ..., Colitta, D. (2000). Effect of long-term treatment with metformin added to a hypocaloric diet on body composition, fat distribution, and androgen and insulin levels in abdominally obese women with and without the polycystic ovary syndrome. J. Clin. Endocrinol. Metab., 85(8), 2767-2774.

Rachoń, D., Teede, H. (2010). Ovarian function and obesity-interrelationship, impact on women's reproductive lifespan and treatment options. Mol. Cell. Endocrinol., 316(2), 172-179.

Rosenfeld, R.L., Bordini, B. (2010). Evidence that obesity and androgens have independent and opposing effects on gonadotropin production from puberty to maturity. Brain Res., 1364, 186-197.

Skałba, P., Dąbkowska-Huć, A. (2005). The metabolic aspects of polycystic ovarian syndrome. Pol. J. Endocrinol., 6(56), 960-963. 